\title{
COMUNICAZIONE SCIENTIFICA IN UNA SOCIETÀ DEMOCRATICA: ASPETTI ETICI
}

\author{
CARLO E. BOTTANI (*)
}

È chiaro che l'etica non può formularsi. L'etica è trascendentale. (Etica ed estetica son uno.) Ludwig Wittgenstein, "Tractatus Logico-philophicus", p. 6.421

Penso che viviamo in un'epoca non scientifica in cui quasi tutti i messaggi delle comunicazioni e della televisione - parole, libri, ecc. - non sono scientifici.

Di conseguenza, c'è una considerevole quantità di tirannia intellettuale in nome della scienza. Richard Feynman, "What is science?"

Il pittore realista. "Fedele in tutto alla natura!"- Ma come ci riesce?/ Quando mai la natura sarebbe risolta in un quadro?! Infinito è il più esiguo frammento del mondo!/Finisce per dipingere solo quello che piace a lui./E che cosa gli piace? Quel che dipingere sa. Friedrich Nietzsche, "La gaia scienza - Scherzo, malizia e vendetta, epigramma 55"

Nature tends to be a wild profusion which our thinking does not fully confine. Nancy Cartwright, "How the laws of physics lie"

SuNTO. - Gran parte della comunicazione e della divulgazione scientifica è pedagogicamente inefficace, anche quella di apparente maggior successo. Nomi come Hawking, Penrose e, più recentemente, Rovelli hanno ottenuto uno straordinario successo di pubblico. Ci sono stati tanti Festival della Scienza e Notti del Ricercatore. Tuttavia serie indagini statistiche mostrano come l'accresciuto livello di alfabetizzazione scientifica produca effetti contraddittori e paradossali. Ciò che veramente manca è la capacità di pensiero critico. Il problema non è solo scientifico, sociologico e psicologico ma anche

(*) Istituto Lombardo Accademia di Scienze e Lettere, Milano, Politecnico di Milano, Dipartimento di Energia, Milano, Italia. E-mail: carlo.bottani@polimi.it 
etico. Molti divulgatori non dicono la verità o non la dicono tutta. Quando ciò accade, la comunicazione, veicolando un'immagine distorta della scienza, può diventare dannosa. Porvi rimedio non è importante solo per la scienza, è importante per la democrazia.

$* * *$

ABSTRACT. - Much of the communication and scientific dissemination is pedagogically ineffective, even that apparently most successful. Names like Hawking, Penrose and, more recently, Rovelli have achieved extraordinary public success. There have been many Science Festivals and Researcher's Nights. However, serious statistical surveys show that the increased level of scientific literacy produces contradictory and paradoxical effects. What really is missing is the capacity for critical thinking. The problem is not only scientific, sociological and psychological but also ethical. Many popularizers do not tell the truth or they do not say it all. When this happens, communication, conveying a distorted image of science, can become harmful. To remedy is not only important for science, it is important for democracy.

In una società sempre più influenzata dalla scienza e dalla tecnologia, la diseguaglianza epistemica è una delle forme gravi della disuguaglianza in generale. ${ }^{1}$ In una visione in cui l'immagine scientifica del mondo è ritenuta essenziale, se non prevalente, si potrebbe pensare che la comunicazione scientifica, dotata di mezzi sempre più sofisticati, possa aiutare a ridurre la disuguaglianza epistemica. Cittadini più informati, e quindi più coscienti e dotati di spirito critico, ${ }^{2}$ sarebbero certamente meglio in grado di valutare, anche sotto il profilo etico, i nuovi risultati della conoscenza scientifica e dell'innovazione tecnologica senza atteggiamenti pregiudiziali, positivi o negativi, anche se Richard Feynman ci ricorda che "le basi fondamentali dell'etica vanno scelte in modo non scientifico". ${ }^{3}$

Intendo qui per "comunicazione scientifica" la trasmissione di informazioni, che ha luogo da parte di esperti ed è destinata a non esperti, sulle conoscenze scientifiche e sulle relative implicazioni tecnologiche, in particolare le più recenti (innovazione). Questo tipo di comunicazione può essere spontanea (divulgazione) o essere commissionata da policy makers (rapporti di consulenza, contenuti per pubblicità sociale ecc.). Le modalità e i contenuti di questa informazione gene-

1 Salvatore Veca, "Il senso della possibilità - Sei lezioni” - Feltrinelli (2018)

2 Wilfrid Sellars, "L'immagine scientifica e l'immagine manifesta", ETS (2013).

3 Richard Feynman, "Deviazioni perfettamente ragionevoli dalle vie battute: le lettere di Richard Feynman” - Adelphi (2006). 
rano responsabilità non sempre facilmente prevedibili quanto a implicazioni e conseguenze individuali e sociali. Ciò ha necessariamente rilevanza etica e i problemi di principio sollevati, trattandosi di innovazione, sono spesso inediti come capita per le conseguenze più tangibili delle nuove tecnologie la cui rilevanza etica è evidente.

Nel caso della pubblicità sociale o, comunque, di una divulgazione pedagogicamente orientata, la modalità comunicativa si è quasi sempre ispirata al cosiddetto "modello del deficit": la comunicazione diverrebbe efficace qualora si colmasse prima, nei limiti del possibile, il deficit conoscitivo specifico del non iniziato. Si può convenzionalmente fissare la data di nascita di questo modello nel 1985 quando il governo britannico pubblicò il "Rapporto Bodmer" per il Public Understanding of Science". Seguirono numerose iniziative per aumentare il grado di alfabetizzazione scientifica dei cittadini. Purtroppo già nel 2002 apparve evidente che il modello produceva effetti contraddittori e paradossali. ${ }^{4}$ Pur essendo, in linea di principio, basato su principi scientificamente e eticamente ineccepibili, esso era già fallito. Nacque allora l'auspicio per un Public Engagement with Science \& Technology. Il rapporto tra esperti e non esperti doveva diventare bidirezionale, interattivo e coinvolgente. John M. Ziman, eminente fisico dello stato solido inglese, lo aveva capito molto prima, anche in relazione a una vera mutazione genetica nel rapporto scienza - società iniziata negli anni 1970. Le iniziative connesse sono ancora in atto ma i risultati rimangono deludenti. Si è infatti trascurato un aspetto essenziale: il progressivo calo di fiducia di una parte consistente del pubblico nei confronti degli esperti (the death of expertise $e^{6}$. Arrivando all'apparente paradosso: maggiore la competenza specifica del non esperto, maggiore la sfiducia negli esperti. ${ }^{7}$ Lidentikit del tipo umano che non ha più fiducia nella competenza certificata non è univo$\mathrm{co}$, ma alcuni tratti comuni si possono individuare. Tralasciando le più frequenti cause personali della sfiducia, si incontra un atteggiamento pregiudiziale e ideologico, non fondato razionalmente e impermeabile alle

4 Vedi gli studi riportati da Nico Pitrelli della SISSA in www.nicopitrelli.it. Molte delle persone più alfabetizzate si rafforzano nei loro pregiudizi antiscientifici.

5 John M. Ziman, "Prometheus bound", Cambridge University Press (1994): uno scritto profetico.

6 Tom Nichols, "The death of expertise", Oxford University Press (2017).

7 Walter Quattrociocchi, Antonella Vicini, "Misinformation”, Franco Angeli (2016). 
argomentazioni scientifiche e tecniche. Una sorta di fede integralista in dogmi antiscientifici enunciati da leader carismatici. Tale fede si rafforza in cerimonie di gruppo celebrate su internet all'interno di gruppi chiusi deve vale il pensiero unico, le cosiddette echo chambers. ${ }^{7}$ Gli adepti di questa fede sono poi insensibili ai fatti ma sensibilissimi alle emozioni che non contrastino con i loro dogmi.

La comunicazione scientifica è così diventata un campo di battaglia in cui si combatte una guerra asimmetrica. Gli esperti combattono, generalmente, in modo tradizionale e i non esperti anti-scientifici praticano una nuova forma di guerriglia sulla rete. Molti esperti avvertono poi uno stato di assedio e cominciano a pensare che il fine giustifichi i mezzi. Si sentono allora in diritto di usare le stesse armi (parlare alla pancia o, comunque, toccare tasti che agiscano direttamente sulle emozioni) per penetrare la cintura protettiva dei rivali (visti come somari) e conquistarne il consenso, ricorrendo di fatto a strumenti che ricordano più la propaganda o la pubblicità (advertising) che la ragione/scienza in una lotta senza quartiere all'eresia. Ritengo bisognerebbe essere invece molto cauti, non solo per forti perplessità di natura etica, ma anche per il rischio di innescare nella comunicazione di massa un circolo vizioso. Anche quando ci si rivolge a non esperti amici privi di pregiudizi antiscientifici, anzi sinceramente interessati alla scienza e al progresso tecnologico.

Questo rischio è particolarmente alto nel caso della divulgazione scientifica intesa in senso stretto anche perché in questo caso l'aspetto etico sembra essere, in prima battuta, meno rilevante: un esperto scrive un articolo o un libro, o partecipa a un documentario o a un'intervista in cui spiega ai non esperti un particolare settore della scienza o della tecnologia. Lo strumento della divulgazione è, in questo caso, un prodotto che deve essere venduto. Che l'autore sia uno scienziato o un giornalista scientifico non fa differenza, se non per l'eventuale notorietà dello scienziato, che favorisce il successo dell'operazione ma aumenta la responsabilità dell'autore. Si richiede anzitutto che il prodotto sia stato redatto secondo gli standard che gli editori ritengono necessari per il successo commerciale e poi che venga adeguatamente promosso. Nel caso delle interviste il format deve adeguarsi nello stile e nei tempi agli assiomi indiscutibili dei professionisti del settore. ${ }^{8}$ La domanda tipica è:

8 Giovanni Carrada, "Comunicare la Scienza - Kit di sopravvivenza per ricercatori”, I quaderni del MDS, 2005. 
"Mi dica in due minuti cos'è il bosone di Higgs". E lo sventurato/a risponde. In una società bombardata da un eccesso di informazioni e dotata di memoria evanescente, passare inosservati è un destino frequente e un buon messaggio che nessuno riceve non è un buon messaggio. I principi etici coinvolti sono quindi in gran parte gli stessi che dovrebbero regolare la qualità dei prodotti e la loro pubblicità rivolta a consumatori che spesso non hanno gli strumenti critici per giudicare nel merito. Essendo la pubblicità necessaria e, in astratto, eticamente neutrale.

Coloro che studiano i fondamenti dell'etica (i filosofi morali) e i giuristi che aiutano il legislatore nella redazione delle proposte di legge e/o le interpretano in qualità di magistrati o avvocati sono esperti ma difficilmente ugualmente esperti di scienza e tecnologia. Se il cittadino non esperto, i cui diritti e doveri sono regolati da leggi che dovrebbero essere ispirate "ab ovo" da principi etici e risultanze scientifiche, non ha più fiducia negli esperti (di ogni tipo) si ha un grave rischio per la tenuta sociale, per la democrazia. Tuttavia un rischio complementare si ha quando il non esperto ha per la scienza, soprattutto per quei settori che sembrano non avere un impatto immediato su ambiente e salute, una sana curiosità, a volte una passione, ma accompagnate da una fiducia acritica che è l'altra faccia della sfiducia. La fiducia o l'assenza di fiducia negli esperti non dipendono, come abbiamo visto, solo dai fatti. Dipendono anche enormemente da come gli esperti comunicano, in base alla loro visione del mondo, a un pubblico spesso irretito in credenze non scientifiche (o in credenze scientifiche ingenue e immotivate) di cui (inconsapevolmente?) gli stessi esperti sono in parte responsabili se hanno adottato strumenti di comunicazione secondo il principio per cui, come già osservato, il fine giustifica $i$ mezzi, apprendo involontariamente la strada anche a chi usa ogni mezzo senza avere la motivazione di alcun fine positivo.

Ritornando al modello del deficit, tra gli scienziati, ma anche tra molte persone colte di formazione umanistica, è ancora assai diffusa l'opinione che lo scarso grado di alfabetizzazione scientifica sia uno dei mali radicali della società attuale da cui discendono due gravi conseguenze: un'opposizione ideologica alla scienza e alla tecnologia da parte di intere fasce della popolazione e la scarsa sensibilità dei politici nei confronti della scienza da cui deriva un finanziamento pubblico del tutto insufficiente. Difficile non essere d'accordo. Da qui però rischia di seguire una certa indulgenza, se non ammirazione, da parte di esperti seri verso divulgatori della stessa disciplina obiettivamente troppo disinvolti, purché rite- 
nuti efficaci. Nuovamente ignorando che, per esempio nel caso di un libro, il numero di copie vendute non misura l'effetto pedagogico, socialmente utile, dell'opera e che il successo commerciale non giustifica superficialità e imprecisioni che possono degenerare in fake news di fatto $\mathrm{o}$, comunque, in una visione della scienza e della ricerca scientifica lontanissima dalla realtà. Multiversi, antimateria, energia oscura sono idee nate nella scienza ma trovano nella rete fantasiose narrazioni. La cosiddetta medicina quantistica, che invece non ha nella scienza alcun diritto di cittadinanza, sulla rete letteralmente imperversa. Altro caso clamoroso quello del computer quantistico. Si tratta di un ambizioso progetto di cui si occupano molti serissimi laboratori scientifici. Da diverso tempo si trovavano in rete annunci che inducevano a pensare (o affermavano) che era già stato realizzato, confondendo simulazioni software di algoritmi quantistici su computer tradizionali con la realizzazione di un nuovo bardware basato su qbit (bit quantistici) fisici in numero sufficiente. Il primo computer quantistico commerciale a 20 bit è invece appena nato e non si sa ancora se potrà svilupparsi in una macchina abbastanza potente da avere un interesse reale e non solo pubblicitario.

Nel caso della cosiddetta singolarità nello sviluppo dell'intelligenza artificiale (AI) la divulgazione si muta poi in fantascienza. La singolarità è prevista da Ray Kurzweil ${ }^{9}$ per il 2029: entro dieci anni le macchine dimostreranno di essere coscienti. Tesi sostenuta, a parte la data, anche da Marvin Minsky, uno dei padri della AI al MIT. Di qui gli scenari apocalittici previsti anche da Stephen Hawking. Fortunatamente voci competenti e molto più equilibrate hanno recentemente demitizzato questi eccessi di futurologia. ${ }^{10} \mathrm{Ma}$ la fantascienza (la forma di pseudo-scienza che piace anche a molti non esperti amici della scienza) attira e vende, così come attira e vende la forma shamanica ${ }^{11}$ di pseudo-scienza che piace a molti non esperti nemici della scienza. Gli estremi si toccano e si fondono. Così lo shamano di turno può anche essere uno scienziato che fa eccessivo appello al fascinoso e al misterioso rivolgendosi agli amici o presunti tali.

9 Ray Kurzweil, "La singolarità è vicina”, Apogeo Education (2008).

10 Meredith Broussard, "Artificial unintelligence", MIT Press (2018) e Marc Mézard, "AI and its limits", Europhysics News, 49/5\&6, 26 (2018).

11 Marco D’Eramo, "Lo shamano in elicottero", Feltrinelli (1999), ringrazio Giorgio Parisi per avermelo segnalato. 
Vale quindi la pena di approfondire il caso, solo apparentemente anodino dal punto di vista etico e sociale, della divulgazione scientifica in senso stretto, particolarmente critico se si divulgano risultati acerbi della scienza, che non andrebbero ancora divulgati senza informare del loro grado di maturazione, o l'ultimo grido dell'innovazione tecnologi$\mathrm{ca}$, generando false aspettative o nascondendo potenziali gravi effetti collaterali (in cauda venenum). L'insuccesso del rapporto Bodmer testimonia quanto sia difficile educare. Diseducare è invece relativamente facile. Non voglio con ciò avallare indiscriminatamente un atteggiamento censorio e moralistico nei confronti della divulgazione ma avverto che questo comincia a comparire sia nella scienza ufficiale, nei confronti della comunicazione scientifica specialistica, ${ }^{12}$ come reazione indignata alla cosiddetta "post-empirical science" (per es. teoria delle stringhe $^{13}$ ) vista come una delle conseguenze/cause della "post-truth society", ${ }^{14}$ sia in una divulgazione scientifica alta e ardua. ${ }^{15}$

Il tema potrebbe essere variamente declinato ma qui voglio concentrarmi su una connessione che ritengo profonda, e sinora non evidenziata, tra la concezione della conoscenza scientifica che l'esperto possiede (talora inconsapevolmente), la sua visione scientifica del mondo, e l'idea di "non esperto" che egli assume come vera, determinandone la modalità narrativa.

Semplificando molto, l'esperto può appartenere a due diverse scuole di pensiero: può essere o non essere un realista scientifico. Per avere un riferimento storico, almeno per chi conosce la storia della fisica, può pensarla come Albert Einstein o come Niels Bohr. Il realista scientifico può anche essere inconsapevole, l'antirealista, o il non realista, no. Oggi sono prevalentemente realisti gli esponenti della Big Science, tra cui spiccano i fisici delle particelle elementari e gli astrofisici/cosmologi. La stragrande maggioranza delle opere divulgative della

12 G. Ellis and Joe Silk, "Scientific Method: defend the integrity of physics", Nature, 516, 321, 2014.

13 Richard Dawid, "String theory and scientific method", Cambridge University Press (2013) The author, a philosopher, claims the unnecessary role of experiments in foundational theoretical physics.

14 L'Oxford Dictionary ha scelto "post-truth" come "word of the year" 1916.

15 Roger Penrose, "Fashion, faith and fantasy in the new physics of the universe", Princeton University Press (2017) tradotto in italiano da Rizzoli con il titolo: "Numeri, teoremi e minotauri: perché la nuova scienza non è affatto scientifica (2017)". 
fisica riguardano questi campi in aperto contrasto con il fatto che il numero di articoli scientifici che appaiono annualmente nel solo settore della fisica della materia condensata è circa il $70 \%$ del totale ${ }^{16}$ mentre il numero di opere divulgative in questo campo è trascurabile.

Per evitare equivoci mi riferisco al caso estremo di un realismo scientifico forte (spesso fondamentalista ${ }^{17}$ ) come può essere vissuto de facto da uno scienziato che non conosce le sottili distinzioni tra i tipi di realismo e quelli di antirealismo descritti da un raffinato filosofo come, per esempio, Hilary Putnam. ${ }^{18} \mathrm{E}$ mi riferisco a un non realismo che non sconfina mai in un anti-realismo a sua volta dogmatico. ${ }^{19}$

Per lo scienziato realista, ${ }^{20} \mathrm{mi}$ riferisco alla fisica per ragioni di competenza, gli elettroni, i neutrini, i quarks, il bosone di Higgs (ecc.) e i campi ad essi associati non solo esistono realmente, a prescindere dall'attuale grado di maturazione della teoria (che descrive un modello) entro la quale sono stati introdotti e definiti, ma hanno tutti lo stesso grado di realtà, lo stesso statuto ontologico di, che so io, un gatto. Inoltre le leggi di natura, cosi come attualmente le conosciamo, sono leggi di validità universale che governano le interazion i tra i componenti della realtà in quanto ne esprimono strutturalmente la verità. Le leggi sono parte della realtà e, in quanto tali, sacre. Se, per esempio, la velocità di rotazione delle galassie a spirale, osservata sperimentalmente, non è in accordo con le leggi della relatività generale, ci si inventa un'ipotetica materia oscura con proprietà inusitate (oscure, appunto) che dovrebbe ammontare a circa il $27 \%$ di tutta la materia. Non sarebbe più sensato (e scientificamente laico) esplorare la possibilità che la relatività gene-

16 Si veda Franco Ciccacci, Il Giornale di Fisica, 57, n. 4, (2016).

17 Adam Becker, "What is real?", Basic Books, 2018 si distingue per superficialità e inesattezza, come ben stigmatizzato nella recensione di Mélanie Frappier su Science, 359, 1474 (2018). Edward W. Kolb per ingenuità in http://videolectures.net/cernacademictraining08_kolb_dudm/\# (2008). Kolb e Becker sono entrambi astrofisici.

18 Hilary Putnam, "La filosofia nell'età della scienza", Il Mulino, 2012.

19 In Il grande disegno, Arnoldo Mondadori Editore (2011) Stephen Hawking e Leonard Mlodinow introducono il "model dependent realism", una forma larvata di non realismo che rischia di essere, di fatto, anti-realista ma viene percepita dal non esperto come realista.

20 Anche per importanti filosofi come per es. Wilfrid Sellars, vedi nota 2, dopo l'inizio del parziale declino nel mondo anglofono del pensiero del grande Ludwig Wittgenstein. 
rale debba essere modificata profondamente e forse, un giorno, sostituita da una teoria migliore $?^{21} \mathrm{Il}$ principale limite di questa particolare visione realista del mondo è la sclerosi: i dogmi scientifici, nati in un particolare contesto storico e culturale ed espressi nel linguaggio di quel contesto, divengono intoccabili. Si potrebbe subito obiettare che esistono realisti scientifici scettici rispetto alle leggi ma non rispetto alle entità di cui sopra (entity realism). Nuovamente però costoro sono filosofi della scienza (per es. Ian Hacking) ${ }^{22}$ e non scienziati praticanti. Inoltre, negando la verità delle leggi, questi filosofi sono sostanzialmente antirealisti. ${ }^{23}$

Sono tra coloro $^{24}$ che ritengono che chi possiede questa visione realista della conoscenza scientifica cada necessariamente in una metafisica dogmatica incompatibile con il metodo scientifico, posto che ne esista uno solo. In altre parole egli, tentato di dire l'indicibile, non si ferma prudentemente sul confine oltre il quale il territorio della metafisica inizia. È convinto, di fatto, che la realtà profonda, quella vera, non sia indicibile e ne fa disinvoltamente argomento della professione. Si riporta allo stadio presocratico di una filosofia che è anche scienza e di una scienza che è anche filosofia e, anche, sacerdozio laico. Ignorando secoli di filosofia e di scienza. Pensa che la scienza possa rispondere con uguale pertinenza sia alla domanda come sia alla domanda perché. Pensa che su temi come spazio, tempo, materia e vuoto basti oggi la parola della scienza che è anche filosofia. Corrispettivamente i filosofi che condividono questa visione si arrendono alla scienza suicidandosi intellettualmente. La filosofia che la pensa così gives up without conditions. È caratteristica conseguenza di questa resa l'accoglienza acritica di stimoli che provengono da settori acerbi della fisica, come la gravità quantistica a loop. ${ }^{25}$ Questa è ancora un interessante insieme di congetture, auto-

21 Giovanni Amelino-Camelia, "Oltre l'orizzonte - Quali nuove frontiere per la fisica", Codice Edizioni, 2017. 1983.

22 Ian Hacking, "Representing and intervening", Cambdrige University Press,

23 Nancy Cartwright, "How the laws of physics lie", Clarendon Press, Oxford, 1983.

24 Forse il primo è Friedrich Nietzsche in "La gaia scienza", aforismi da 110 a 113,1882

25 Carlo Rovelli e Francesca Vidotto, Covariant Loop Quantum Gravity, Cambridge university Press, 2014. 
proclamatesi teoria senza ancora il conforto di dati sperimentali, che non contiene la variabile tempo nella sua arzigogolata formulazione matematica. Ed ecco che alcuni filosofi si affrettano a preoccuparsene filosoficamente, sprecando quel poco tempo, irreversibile, che hanno a disposizione. Si badi che non sostengo affatto che le visioni fisiche di spazio, tempo, materia, vuoto ecc. non debbano essere considerate dai filosofi. Albert Einstein, a partire dal 1922 (anno in cui gli fu conferito il premio Nobel 1921 per la teoria dell'effetto fotoelettrico) aveva invece dalla sua, sin dal 1919, alcune verifiche sperimentali per la teoria della relatività generale. Tuttavia cominciò a dimostrare una sorta di disprezzo per il tempo dei filosofi, in particolare per le concezioni di Henry Bergson, dichiarando in un dibattito pubblico a Parigi che "il tempo dei filosofi non esiste" e che il solo tempo obiettivo è quella della fisica (la sua). Si veda a proposito l'interessantissimo saggio di Jimena Canales. ${ }^{26}$

Da un punto di vista storico poi, la fisica moderna ha avuto l'enorme successo che sappiamo e ha prodotto la rivoluzione tecnologica che viviamo perché ha seguito Niels Bohr (un non realista scientifico), a dispetto di chi sostiene, come Murray Gell-Mann, che "Bohr ha fatto il lavaggio del cervello a due generazioni di fisici" ${ }^{13}$. Bohr sosteneva che: "Non c'è un mondo quantistico. C'è solo una descrizione fisica astratta. È sbagliato pensare che il compito della fisica sia scoprire l'essenza della natura. La fisica riguarda ciò che della natura possiamo dire" e "Il nostro compito non è penetrare l'essenza delle cose, di cui peraltro non conosciamo il significato, ma sviluppare concetti che ci permettano di parlare in modo fruttuoso dei fenomeni naturali". ${ }^{27}$

Oggi lo scienziato realista che si rivolge al non esperto ha un enorme vantaggio comunicativo sul non realista. Egli condivide con il non realista la consapevolezza che l'attuale descrizione scientifica del mondo si basa necessariamente su teorie matematiche complesse e antiintuitive $e^{28}$ e che questo stato di fatto è forse la principale ragione della

26 Jimena Canales, The physicist and the philosopher, Princeton Press, 2015.

27 Da una lettera di Niels Bohr a H.P.E. Hansen del 20 luglio 1935; citato in Abraham Pais, Ritratti di scienziati geniali, Bollati Boringhieri, 2007, p. 37.

28 James Cushing, scrive in 'Models and Methodologies in Current Theoretical High Energy Physics', Synthese 50 (1982), p. 78: "When one looks at the succession of blatantly ad hoc moves made in QFT [Quantum Field Theory] (negative-energy sea of electrons, discarding of infinite self energies and vacuum polarizations, local gauge invariance, forcing renormalization in gauge theories, spontaneous symmetry breaking, 
distanza epistemica che lo separa dal non esperto, che separa l'esoterico dall'essoterico. Ma egli crede che la realtà non è come ci appare. ${ }^{29} \mathrm{La}$ realtà vera è colta dalle sue complesse teorie, i dogmi della sua religione laica che i profani colgono come affascinante mistero, tanto più affascinante quanto più misterioso: egli sta penetrando a fondo nel reale conoscendo scientificamente quelle che Kant chiamava le cose in sé e che riteneva si potessero solo pensare (Wittgenstein riteneva che il mistico potesse solo mostrarsi). Ciò che appare può essere ricondotto a ciò che non appare: il gatto agli elettroni ecc. Ricondotto: la parola magica.

Il riduzionismo microscopico è per lo scienziato realista una necessità logica come il ricorrere agli atomi era essenziale per la filosofia/scienza di Epitteto e Democrito. Sappiamo oggi bene che il riduzionismo è uno strumento concettuale irrinunciabile anche per lo scienziato non realista ma, per quest'ultimo, è un puro strumento concettuale di cui riconosce vantaggi e svantaggi. Il centro di massa di un sistema di particelle non esiste, ma è un indispensabile strumento di semplificazione concettuale. Il riduzionismo ha poi limiti intrinseci di fronte alla complessità e all'emergenza connessa alla rottura spontanea di simmetria. ${ }^{30}$ Ignorare questi limiti genera l'arroganza stigmatizzata dal premio Nobel Philip Anderson: "I fisici, e gli scienziati in genere, amano fare due cose; (a) smontare, analizzare riducendo a semplici componenti; (b) mistificare, dire: non è semplicemente questo, è quello; quello che vedi non è quello che c'è. Lo scienziato si è arrogato il ruolo dello sciamano o del mullah. Tutto proviene da una Causa Prima - la Prima Equazione - e solo lui può investigare la natura con le sue costosissime apparecchiature e comprenderla con le sue astruse teorie. L'arroganza nutrita da questa attitudine mentale è tale che, solo sperimentandola, si può credere che possa esistere." ${ }^{31}$

permanently confined quarks, color, just as examples) and of the picture which emerges of the 'vacuum' (aether?), as seething with particle-antiparticle pairs of every description and as responsible for breaking symmetries initially present, one can ask whether or not nature is seriously supposed to be like that."

29 Carlo Rovelli, "La realtà non è come ci appare", Raffaello Cortina Editore, 2014. In "Sette brevi lezioni di fisica" (Adelphi 2014) Rovelli afferma che la "realtà è interazione" (tra che?) lasciandoci nel dubbio riguardo alla sua posizione che, altrove (anche nello stesso libro), sembra molto più orientata al realismo scientifico, anche in assenza di conferme sperimentali.

30 Philip Anderson, "Basic Notions of Condensed Matter Physics", Addison Wesley (1984).

31 Philip Anderson, "More and different", World Scientific, 2011. 
Ecco che allora il realista colma/annulla, quasi sempre con successo di pubblico, la distanza epistemica che lo separa dai profani non fornendo conoscenze (che il profano non potrebbe comprendere) ma metafisiche emozioni. È un fatto che si verifica sempre più frequentemente nella divulgazione, non una necessità logica che discende dalla sua visione del mondo. Chi non si emoziona contemplando il mistero del cielo stellato in cui si nascondono minacciosi buchi neri? Chi non si emoziona apprendendo che, in una delle versioni della gravità quantistica, "il tempo non esiste", mentre in altre versioni esistono particelle supersimmetriche che popolano un supermondo? Nell'attuale teoria quantistica dei campi, dove i campi e le loro simmetrie hanno scalzato le particelle dal ruolo di protagonista, Platone sta, ritengo sfortunatamente, prevalendo su Aristotele. Un'idea astratta di bellezza e di naturalezza guida dal 1970 la fisica teorica fondativa, dimentica del ruolo essenziale dei fatti empirici ${ }^{32}$ e dei limiti dei suoi modelli.

Lo scienziato realista che divulga si trova a poter sfruttare una quasi perfetta coerenza tra domanda e offerta. Per rispondere alla richiesta di certezze che proviene dalla società liquida, confusa e disorientata, dove spesso si vive una vita grigia non salvata dalla bellezza, egli ha già un'affascinante risposta pronta: come resistere alla tentazione? Ciò che funziona nel cinema perché non dovrebbe funzionare nella divulgazione? "Io ho viste cose che voi umani non potreste immaginarvi: navi da combattimento in fiamme al largo dei bastioni di Orione e ho visto i raggi B balenare nel buio vicino alle porte di Tannhäuser". ${ }^{33}$ $\mathrm{E}$, infatti, funziona, anche quando si presenta una congettura come fosse una teoria consolidata. E lo shamano ricompare, travestito da scienziato.

Detto più brutalmente, si usa il vecchio metodo, profondamente non etico, di épater le bourgeois, rivelatore della sostanziale disistima che l'esperto ha del pubblico. Sappiamo quanto sia oggi pericoloso convincere usando le emozioni in luogo dei fatti. Una scienza che, per conquistarsi il pubblico, usa tecniche di advertising basate su una previa analisi psicologica e di mercato.

Anche lo scienziato non realista rischia di comunicare in modo

32 Sabine Hossenfelder, "Lost in math - how beauty is leading physics astray", Basic Books (2018).

33 Dal finale del film "Blade runner" diretto da Ridley Scott, 1982. 
eticamente scorretto. In più, non potendo fare un facile appello alle emozioni, rischia l'inefficacia. Egli è normalmente un pluralista attratto dal fallibilismo di Peirce ${ }^{34}$ e dal convenzionalismo che va da Poincaré35 a Bohr. Da Bohr ha imparato poi ad accettare l'incoerenza interna di teorie che funzionano e una certa dose di opportunismo che lo allontana molto dal mito dell'eroe romantico in cerca del brivido che deriva dal contatto con l'essenza ultima delle forze della natura. MerleauPonty, riflettendo sul significato della meccanica quantistica nella forma oggi considerata ortodossa, scrive: "Possiamo avere la tentazione di liberarci del problema della verità. La fisica non dovrebbe essere concepita come una ricerca della verità, dovrebbe rinunciare a determinare un reale fisico: essa sarebbe soltanto un sistema di misure, collegate fra loro mediante equazioni, che permetterebbero di prevedere il risultato delle misurazioni future. La fisica formalista riceve ogni libertà, ma perde il suo contenuto ontologico. Non esprime nessun modo di essere, nessuna realtà". ${ }^{36}$

Si capisce quindi la critica di Husserl alla scienza occidentale, come ho già scritto nel mio libro "Il mestiere della scienza" ${ }^{37}$ Edmund Husserl, il maestro di Heidegger, nel 1935 inizia a pubblicare un testo dal titolo significativo "La crisi delle scienze europee e la fenomenologia trascendentale". Crisi delle scienze? Sembra intrinsecamente strano detto alla fine del periodo d'oro della fisica moderna. Ai tempi di Husserl la società certo stava vivendo una profonda crisi di identità, come oggi. Una gravissima crisi economica era ancora sensibile. Come oggi. Ma le scienze dure, come ho appena ricordato, avevano già ottenuto magnifici nuovi risultati, sia sul piano teorico sia su quello delle ricadute tecnologiche. Perché parlare di crisi? Il fatto è che Husserl vede in profondità sotto la superficie un limite che è tipico della versione non realista della scienza. Husserl sostiene: "L'arte di inventare sempre nuovi procedimenti simbolici, la cui razionalità è appunto di ordine meramente simbolico [...], senza alcun tentativo di comprensione evidente, viene praticata in modo sempre più perfetto. [...] e così le scien-

34 Charles Sanders Peirce, "The Scientific Attitude and Fallibilism," in Justus Buchler, ed., Philosophical Writings of Peirce. Dover (1955).

35 Jules Henri Poincaré, "La scienza e l'ipotesi”, Bompiani (2003).

36 Maurice Merleau-Ponty, La Natura, Raffaello Cortina Editore, 1996.

37 Carlo E. Bottani, "Il mestiere della scienza - La ricerca scientifica tra artigianato e Big Science”, Franco Angeli (2015). 
ze diventano quello che sono, fabbriche di proposizioni praticamente utili, in cui si può lavorare come operai o come tecnici scopritori, a cui, in veste pratica, si può attingere anche senza un'intima comprensione, cogliendo, nel migliore dei casi, semplicemente la razionalità tecnica." La scienza moderna è estremamente utile ma è anche disancorata dai bisogni psicologici dell'uomo. Non può sostituire la filosofia, non può direttamente orientare le decisioni politiche, non può surrogare la religione di chi è tradizionalmente religioso né potrebbe diventare, di fatto, la nuova religione di chi religioso, in senso tradizionale o meno, non è. E poi, orrore!, è fallibile e in perenne mutamento.

Se questa concezione della scienza venisse oggi sbrigativamente comunicata senza contestualmente mostrare/dimostrare come la relativa incertezza epistemica sia sì strutturale ma assolutamente tollerabile, anzi creativa, e senza sottolineare gli indubbi vantaggi che la scienza e la tecnologia hanno portato all'umanità, il rischio di aumentare la sfiducia negli esperti sarebbe enorme (gli esperti delegittimerebbero se stessi). Non potendosi basare sulle emozioni per produrre interesse/fiducia si rischierebbe di produrre ulteriori emozioni negative che condurrebbero a un pessimismo acritico e alla mancanza di fiducia, inoculando dubbi di fede anche negli adoratori della scienza, cioè in coloro che hanno aderito con entusiasmo alla metafisica dogmatica del realismo scientifico. Si contribuirebbe così a produrre versioni devianti di principi precauzionali che diventerebbero completamente paralizzanti.

Lo scienziato non realista, conscio di questi rischi di procurato allarme, ha quindi il dubbio morale se non sia meglio dire solo "la mezza messa" per non essere male interpretato da chi non ha sufficienti strumenti concettuali: fingersi realista per un bene superiore, autocensurandosi.

In verità, una modalità di comunicazione efficace e sincera, basata su un'emozione autentica e profonda, primordiale, è disponibile anche per lui. Ricordare che "Non come il mondo è, è il mistico ma che esso è" ${ }^{38}$ Se il mondo contenesse uno spazio piatto e non curvo, se non vi fossero buchi neri e materia oscura, se fosse eterno e non nato dal bing bang ecc., dovrebbe comunque essere per noi avvincente constatare che, anzitutto, un mondo su cui si affacciano i nostri occhi esiste. Partendo da questa emozione prima, l'immenso spazio per emozionarsi

38 Ludwig Wittgenstein, “Tractatus Logico-Philosophicus”, proposizione 6.44. 
attraverso la narrazione di come è il mondo rimane inalterato, non dimenticando mai che, mentre i fatti (e le cose) non dipendono dal linguaggio, ciò che diciamo (anche scientificamente) dei fatti (e delle cose) dipende dal linguaggio. ${ }^{39}$

Ma forse continuo a preoccuparmi solo del rischio che oggi corrono le nostre società democratiche, in un mondo che sta per essere dominato da potenze ben diverse. I grandissimi fisici russi dell'era sovietica (Landau, Davidov ecc.) trovarono un modus vivendi con il potere del loro tempo anche se furono costretti a scrivere le introduzioni dei loro magnifici testi di meccanica quantistica in modo da non far capire al regime che la pensavano, sostanzialmente, come Bohr (Landau era stato da Bohr a Copenhagen nel 1929).

"Although we do not consider in this book special methodological problems, the exposition is based upon dialectic materialism, that is, we start from the idea that the regularities of atomic and nuclear physics which are studied in quantum mechanics are objective regularities of nature". ${ }^{40}$

La frase di Davidov è, in realtà, astutissima. Dichiarando la fede nel materialismo dialettico si mette al sicuro. Ma tutto quanto segue potrebbe ugualmente bene essere dichiarato da un non realista.

Torno qui alla premessa: non realista, non antirealista. Cioè un ricercatore scientificamente laico che, intrinsecamente scevro da ogni arroganza, ma forte delle sue buone ragioni non dogmatiche, non considera i non esperti dei minus habens miscredenti da convertire alle certezze della religione della scienza in cambio delle perline colorate di rutilanti emozioni. Riguardo al realismo scientifico egli è semplicemente agnostico. In questa assenza di arroganza sta la chiave per intravedere la soluzione per una corretta comunicazione scientifica: l'atteggiamento di fondo che, a mio parere dovrebbe guidare l'esperto che si rivolge, con competenza e rispetto, al non esperto.

Sia il realista scientifico (che potremmo definire metafisico/mistico) sia l'antirealista scientifico, che potremmo qui chiamare scettico, non si attengono al metodo scientifico. Infatti anche affermare che gli elettroni, i neutrini, i quarks, il bosone di Higgs (ecc.) e i campi ad essi

39 Willard van Orman Quine, "Parola e Oggetto", Mondadori, Milano (1970).

40 A.S. Davidov, "Quantum Mechanics", Pergamon Press 1965 (tradotto da D. ter Haar dall'originale Russo del 1963). 
associati non esistono non ha significato scientifico, è un flatus vocis. Cosa si intenda quando in fisica si dice elettrone è definito operativamente all'interno di una precisa prassi sperimentale e formalizzato in un modello matematico che consente di prevedere fatti osservabili e misurabili. Di più non serve anche se di più sarebbe affascinante. Oggi è possibile vedere in laboratorio gli atomi con il microscopio a effetto tunnel, non è invece possibile vedere i singoli elettroni all'interno degli atomi. Potrei forse dire che gli atomi esistono e gli elettroni no? Sarebbe grottesco. A Sellars ${ }^{2,41}$ si potrebbe oggi forse obiettare che il confine tra l'immagine scientifica e l'immagine manifesta del mondo è un confine mobile e sfumato dal progresso della strumentazione al punto da indebolire la sua distinzione fondamentale tra le due.

A questo proposito, due anni fa mi convinsi che sarebbe stato interessante studiare un possibile parallelo Bohr-Wittgenstein. Non sapevo fosse già stato fatto da quattro anni (!) e non da un filosofo ma da uno stimatissimo collega fisico teorico, Stig Stenholm. ${ }^{42}$ A mio modo, non seguo qui Stenholm da vicino, uso Wittgenstein per cercare di chiarire le considerazioni precedenti. Wittgenstein critica sia il metafisico sia lo scettico.

$\mathrm{Al}$ metafisico (qui il realista scientifico) Wittgenstein, come ho già anticipato, ricorda che: "Non come il mondo è, è il mistico, ma che esso è" e che "Tutta la moderna concezione del mondo si fonda sull'illusione che le cosiddette leggi naturali siano le spiegazioni dei fenomeni naturali" (ibidem, proposizione 6.371). Allo scettico (qui l'anti-realista scientifico) ricorda che: "D'una risposta che non si può formulare non può formularsi neppure la domanda, L'enigma non v'è. Se una domanda può porsi, può pure avere una risposta. Lo scetticismo è non inconfutabile, ma apertamente insensato, se vuol mettere in dubbio ove non si può domandare. Perché dubbio può sussistere solo ove sussiste una domanda; domanda solo ove sussiste una risposta; risposta, solo ove qualcosa può esser detto. (ibidem, 6.5-6,51). A entrambi ricorda che: "Noi sentiamo che, anche una volta che tutte le possibili domande scientifiche hanno avuto risposta, i nostri problemi vitali non sono anco-

41 In soldoni: Sellars definisce l'immagine scientifica quella che richiede la postulazione di entità invisibili.

42 Stig Stenholm, "The quest for reality: Bohr and Wittgenstein - two complementary views", Oxford (2011). 
ra neppure toccati. Certo allora non resta più domanda alcuna; e appunto questa è la risposta." (Ibidem 6.52).

I nostri problemi vitali non sono ancora neppure toccati! La scienza non deve mai essere ispirata dalla tracotanza. Come si riconquista la fiducia? Cominciando a dire con equilibrio la verità e basandosi sui fatti. E la verità è che una concezione monista di una scienza a rischio di arrogante scientismo non è sostenibile così come non lo è una concezione analoga dell'etica. Ma l'etica, per formulare principi utili all'uomo, affinché l'innovazione scientifica e tecnologica non producano effetti collaterali ingovernabili, ha bisogno di avere accesso alle migliori conoscenze scientifiche disponibili essendo in grado di comprenderle. Sono anzitutto i filosofi morali a doversi poter fidare della scienza. Ciò richiede che gli scienziati, senza perdere una giustificata autostima, non dimentichino il senso del limite. Giovanni Amelino-Camelia, nel suo interessante libro "Oltre l'orizzonte", ${ }^{21}$ ci ricorda che Albert Einstein tra il 1905 e il 1917 "produsse una serie di impressionanti scoperte e teorie, attraverso un approccio curioso e umile nei confronti della natura[, sempre guidato dall'esigenza di spiegare fatti sperimentali,] mentre negli anni successivi ne adottò uno più arrogante" e non fece più scoperte della stessa eccezionale rilevanza. ${ }^{43}$ Spero che alla fine di queste considerazioni non stupisca il fatto che il mito del genio trascurato e un po' anarchico che tutti conosciamo, e che è assurto a un grado di popolarità sinora ineguagliato in campo scientifico, corrisponde all'ultima parte del secondo periodo, escludendo però gli ultimi due, tre anni. Poco prima di morire, ci ricorda Jimena Canales, Einstein confidò all'amico Michele Besso, che sempre aveva sostenuto in privato con lui le ragioni di Bergson, di cominciare a dubitare. Come ogni scienziato veramente grande stava considerando seriamente la possibilità di avere avuto torto, almeno nella sua monolitica concezione del tempo. Solo quegli scienziati che, contemplando la possibilità di avere torto, scendono dall'altare della loro indispensabile competenza e si mescolano con la gente comune, possono sperare di riconquistarne la fiducia. Impresa difficile ma necessaria e giusta.

43 Anche se Amelino Camelia sembra ignorare gli importanti contributi di Einstein dopo il 1917, la tesi dei "due Einstein", pur essendo troppo schematica, ha un fondo di verità che si può trovare molto meglio documentato nel saggio di Jimena Canales (ibidem). Penso che i due Einstein non si siano succeduti nel tempo, ma siano sempre coesistiti nell'unico Einstein storico con peso relativo alterno. 
A questo proposito fa molto pensare una frase di Richard Rorty: "Essi [i pragmatisti] pensano che le sole virtù delle quali gli scienziati dispongono sono la tendenza ad affidarsi alla persuasione piuttosto che alla forza, il rispetto delle opinioni dei colleghi, la curiosità e il desiderio di nuove acquisizioni e nuove idee. I pragmatisti non credono all'esistenza di una virtù intellettuale, la «razionalità», al di là e al di sopra di queste virtù morali." ${ }^{\prime 4}$

$\mathrm{Ma}$, attenzione: "[...] con un tono vagamente rortyano (Gianni Vattimo $)^{45}$ ci assicura che in etica contano i discorsi edificanti. Non si dice ad altri: 'ti mostro che...' Si deve dire loro, piuttosto: 'non ti pare che sarebbe meglio se...?'. Ma la manovra nasconde il vecchio trucco. Sarebbe meglio, perché?" ${ }^{4}$

44 Richard Rorty, "Scritti filosofici vol. 1", Bari Laterza (1994).

45 Il corsivo è mio.

46 Salvatore Veca, La barca di Neurat, Edizioni della Normale, Pisa (2015). 